\title{
Về bàn phím máy tỉnh cho chữ Việt
}

\author{
Ngô Trung Viêt, James Do \\ Ngô Thanh Nhàn \& Hoàng Công Thành \\ Viện Công nghệ thồng tin
}

\section{Các nguyên tắc xây dựng bàn phím chữ Việt}

Bàn phím chữ Việt cân phải đáp ứng một số đòi hởi có tính chất nguyên tắc phù hợp với quan điểm chung về phát triên công nghệ thông tin ở Việt Nam. Các quan điềm chinh của việc phát triền công nghệ thộng tin ở Việt Nam là:

- Quan điêm hệ thống mở, dựa trên cơ 'sờ chuân được lựa chọn phù hợp với xu thế tiến hoá của công nghệ thông tin trên thế giới. Quan điêm này đưa tới việc cấu tạo bàn phím chữ Việt cần tuân thư theo các chuân quốc tế hiện có về thông tin.

- Phát triên công nghệ thông tin ở Việt Nam trong giai đoạn hiện nay nhằm vào các ứng dụng thông tin ở Việt Nam trong mọi hoạt động kinh tế, xã hội và văn hóa. Diều này yêu cẩu bàn phím chữ Việt phải phán ánh được đặc thù của.chữ Việt và bảo đảm khả năng biêu dièn đầy đỉ cho chữ Việt đồng thời phải đủ đơn giản để mọi người bỉnh thường dề dàng dùng được chữ Việt trên máy tính.

Các quan điềm có tính chất nguyên tắc trên quy lại thành các yêu cầu cơ bản đối với việc thiết kế bộ bàn phím chuẩn chữ Việt là:

- Tính đây đủ: tức là cách bố trí và cách tồ hợp các phím có trên bàn phím "phải biểu diễn được cho mọi hỉnh chữ Việt.

- Tính nhất quán: tuân thư các yêu câu của các chuần quốc tế khi cấu tạo bàn phím và cácb mã hóa kí tự. 
- Tính tương hợp: dùng được chữ Việt cùng với chữ Anh trên các loại bàn phím phổ biến. Dặc biệt, yêu cẩu này dấn tới việc cẩn có cờ báo hiệu bàn phím đang ớ trạng thái tiếng Việt hay tiếng Anh.

- Tính dễ sử dụng: đề cho đại đa số người Việt có thề dùng được công nghệ thông tin, bàn phím chữ Viet cần phải có kiến trúc thỏa đảng, gẩn với thói quen viết của người Việt.

\section{Cài đặt bàn phím chữ Việt: các mức logic và vật lí}

Bàn phím là công cụ con người dùng đề đưa thông tin vào máy tính. Bàn phím cho ngôn ngữ nào cũng phài phản ánh được đặc thù của ngôn ngữ đó. Nói riêng bàn phím phải cho phép đưa vào máy tính mọi con chữ có trong bảng chũ cái của ngôn ngữ. Tiư yêu cầu về tỉnh tương hợp rõ ràng rằng bàn phím chữ Việt cần dựa trên kiến trúc có sẵn của các bàn phím phồ biến trên thế giới. Vấn đề thiết kế riêng một bộ bàn phím cho chữ Việt chưa được xem xét tới ở đây do không đáp ứng được yêu cầu tương hợp.

Theo chuẩn ISO và ECMA-115 về các bàn phím quốc tế, mọi bàn phím đều được phân chia thành các mức độ cài đặt logic và vật lí. Do vậy việc xây dựng bàn phím chữ Việt cũng cần tu ân thủ nguyên tắc này. Nhiệm vụ cơ bản của mọi bộ bàn phím là chuyên tín hiệu do người sự dụng tác dộng trên bàn phím thành các mã hiệu ứng với một đơn vị cơ sở trong bảng mã của ngôn ngữ. Vậy mức độ cài đặt logic cho bàn phím xác định quy tắc đặt tương ứng giữa các đơn vị cấu thành bằng chữ cái của ngôn ngữ với các phỉm logic của bàn phím. Mức độ cài đặt vật lí cho bàn phím xác định cách bố trí cụ thể các phím trên bàn phím .

Bàn phím QWERTY là một cách thề hiện cho bộ mã ASCII 7 bit, trong đó mọi kí tự hinh chữ trong bộ mã này đều được đặt tương ứng với một phím bấm hoàn toàn xác định. Bàn phím AZERTY là một cách cài đặt cho bộ mã ASCII mờ rộng để tả được cho mọi chữ Pháp. Đề xây dựng bàn phím cho chữ Việt với tiên đê xứ dụng các loại bàn phím QWERTY hay AZERTY có sẵn, cũng cần xác định ra cách cài đặt logic và vật lí cho bàn phím chữ Việt trên nên những bàn phím này.

Cách cài đặt logic cho bàn phím chữ Việt sẽ xác định ra cách phân tích các đơn vị chính tà chữ Việt thành các thành phận biểu diên được theo bàn phím mã hoá theo ASCII 7 bit. Cách cài đặt logic này cũng còn bao gồm việc tạo lại mã đúng cho kí tự chữ Việt từ các tín hiệu thu được trên bàn phím do người dùng đưa vào. Mặt khác, cách cài đặt logic còn bao gồm cả việc tái tạo lại hỉnh dáng đích thực của chữ Việt có mang dấu thanh thề hiện trên màn hỉnh.

\section{Dặc thù cấu tạo cách viết tì̀ tiếng Việt}

Theo TCVN 5712 thì bảng chữ cái tiếng Việt bao gồm 33 chữ cái, thêm 7 chữ cái mới nếu so với số các chữ cái trong bảng chữ tiếng Anh: 


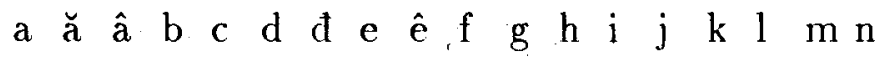

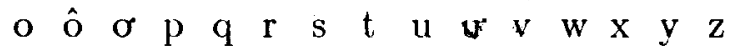

(các chữ cái mới thềm vào là: ă, $\mathrm{a}, \mathrm{d}, \hat{\mathrm{e}}, \hat{o}, \sigma, u ̛$ ). Ngoài ra, tiếng Việt còn có thêm 6 thanh với 5 thanh có mang cách kí hiệu riêng:

dấu huyển, dấu hỏi, dấu ngã, dấu sắc, dấu nặng.

Như vậy so với bảng chữ rái tiếng Anh thì bảng chữ cái tiếng Việt có nhiều hơn 12 thành tố. Điều này cûng có nghĩa là không thề tồn tại tương ứng một giữa hai bảng chừ Việt - Anh. Nói cách khác, chỉ riêng các bàn phím hiện có cho tiếng Aúnh không đủ để diên tả cho cái chữ cái trong bảng chữ tiếng Việt, nếu không đưa ra một cách thức nào đó biêu diễn cho một chữ Việt bằng tồ hợp của vài phím trên bàn phín cho tiếng Anh. .

Theo cách viết tay quen cula đại đa số của người Việt Nam thì trong một từ, tất cả các chữ latinh cơ bản được viết ra trước hết. Sau đó các dấu trên nguyên âm và dấu thanh mới được bồ xung thêm đề tạo nên dúng chữ Việt. Như vậy trong cách viết chữ Việt đã có sự phân tích các chữ cái Việt thành tồ hợp của các chữ cái latinh và các dấu nguyên âm, dấu thanh. Con chữ đúng trong bảng chữ Việt không được tạo ra ngay khi viết mà phài đợi đến giai đạon cuối cùng, bồ xung thêm các dấu phụ thì mới thành hình. Các dấu phụ này bao gồm dấu trên nguyên âm của các nguyên âm thuần Việt và dấu thanh. Theo quan điềm cách viết như vậy có ba loại dấu nguyên âm: dấu mũ của â, ê , ô, dấu mũ ngược của ă và dấu râu của ơ, ư. Đặc thù này dẫn tới một số cách phân tích chữ Việt thành các thành phẩn nhỏ hơn, có thể biều diễn được theo bộ mã ASCII. Tuy nhiên theo quan điềm này ta thiếu mất sự nhất quán với cấu tạo bảng chữ cái tiếng Việt, trong đó đơn vị nhỏ nhất của bảng chữ là các chữ cái, không có sự phân tích nhỏ hơn.

Giải pháp chinh thống theo cấu tạo của bảng chữ Việt dẫn tới việc phải bồ xung thêm 7 phím logic cho 7 nguyên âm thuân Việt và 5 phím logic cho 5 dấu thanh. Do yêu câu phài sừ dụng được bàn phím chữ Việt trên các bàn phím phổ biến hiện tại, QWERTY và AZERTY, nên 12 phím logic trên phái được bố trí thêm vào cấu trúc hiện tại của bàn phím có sãn. Giải pháp không chính thống dựa theo cách viết thông thường chữ Việt đưa tới cảch bố trí các phím có sãn của bàn phím phồ biến kiêm luôn chức năng của các dấu nguyên âm và dấu thanh: cân tối thiêu 7 phím mang ngữ nghĩa đặc biệt. Cá hai giải pháp này đều dẫn tới việc tiến hành các xứ lí chặn bắt tín hiệu gõ vào từ bàn phím để biến đồi thành mã cho chữ Việt và xư lí hiện thị chữ lên màn hình. Nói cách khác, đêu phải tiển hành mã hóa và giải mã cho việc tạo kí tự Việt theo các mã của bàn phím, mã ASCII.

Trong trường hợp đã có cách biêu diễn chữ Việt bằng một số tồ hợp phím thông lhrờng. dù chính thống haỵ không, thì vấn đề nữa cân phài xem xét tới là cấn có 
cờ báo hiệu trạng thái bàn phím đang ở trong chế độ gô tiếng Việt hay tiếng nước ngoài. Thực chất vấn đề là ở chố chúng ta đã làm quá tải bàn phím QWFRTY hay AZERTY đề có thể hiều diễn cho chữ Việt. Do đó, dề phân biệt cách thông dịch tín hiệu được gõ trên bàn phím, cân phải có dấu hiệu nào đó của hệ thống xác định chế độ làm việc hiện tại của bàn phím đang trong ngôn ngữ nào. Đây là một tín hiệu thông báo cho cả hệ thống lẫn người dùng cho nên nó phải vì̛a được quy định ở mức mã hiệu hệ thống lẫn mức cài đặt nhận biết được cho người dùng.

\section{Mã hóa cách tạo chũ̃ Việt tì̛ bàn phím quốc tế (bàn phímlogic)}

\subsection{Giải pháp chính thóng}

Giải pháp chính thống dựa trên bảng chữ cái tiếng Việt: mã hóa các đơn vị chính tá Việt theo các phím trên bàn phím quy ước. Xuất phát từ bảng chữ tiếng Việt và so với bảng chữ tiếng $A n h$, các đơn vị chính tà Việt có thêm là:

$\breve{a}, \hat{a}, d, \hat{e}, \hat{o}, \sigma, u$, dấu huyển, dấu höi, dấy ugã, dẩy sắc, dấu nặng

Trong TCVN 5712, các đơn vị chính tả này đã được gán cho các mà là

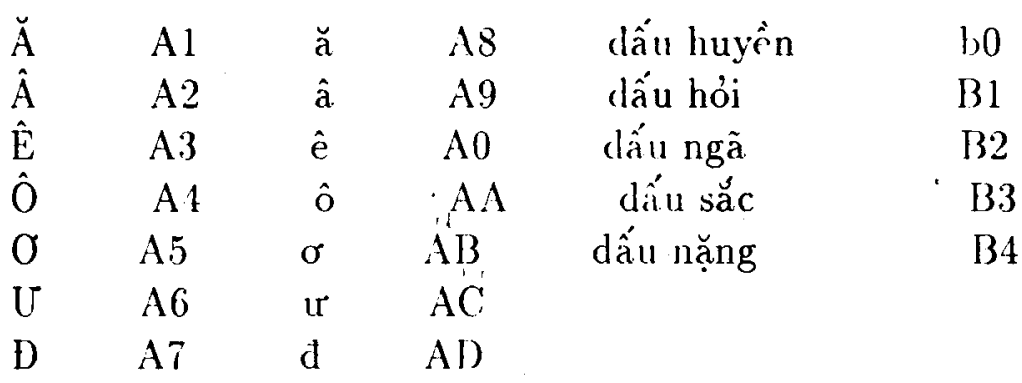

Vấn đê còn lại là thiết lập tương irng giữa các đơn vị chỉnh tà này với một phím logic của bàn phỉm. Diền xıất phát cuia ta là từ bàn phím quốc tế phổ biến, mọi vị trí có sằn đêu đà được gán cho một kí tự của bộ mà ASCII 7 bit theo các mức cài đặt bậc 1 và 2 . Nay cùng bàn phím dó còn phài thề hiện thêm các chữ tiếng Việt, cho nên tương ứng giữa các đơn vị chính tả Việt với các bàn phím logic chì có thề thực hiện được thông qua việc làm quá tải phím bấm. Điều này có nghia là dùng các phím cũ nhưng bồ xung thêm các chức năng mới. Các chức năng mới có thề được cài đặt ở các mực từ 1 tới 4 . Do yêu cầu đơn giàn trong xừ dụng bàn phím chữ Việt, các phím được làm quá tải này cần giữ vai trò phím sư dụng chính cho đơn vị chính tà Việt, tức là các chức năng trước đây cuia chúng cấn được chuyền sang mức cài đặt 3 hay 4 cho bàn phím, nhường mức cài đặt 1,2 cho chữ Việt.

\subsection{Giaii pháp không chính thóng}

Giải pháp không chính thống dựa trên cách phân tích các đơn vị chính tà Việt thành các thành phẩn nhỏ hơn, mã hơa được theo các phím của bàn phím ASCII. 
Khi đó sẽ không có các phím tương ưugr với các đơn vị chỉnh tả Việt, tức là người sư dụng bắt buộc phải nhớ cách mã hó chữ Việt theo kiểu tạo chữ đề gõ vào máy. Diều đó dî nhiên là một nhược điểm khó sư dụng của giải pháp kí thuật này đối với đa số người sư dụng thông thường. Tuy nhiên một khi đã dùng quen thì nó lại tó ra rất tiện lợi và nhanh chóng.

\subsubsection{Kiêu bộ mã ASCII 7 bit}

Cách mã hóa dựa trên bảng mã ASCII 7 bit thực hiện mã hóa các chữ Việt theo chữ cái latinh, dấu nguyên âm và dấu thanh được mã hóa độc lập nhau, dựa trên các kỉ hiệu có sẵn hay gân giống về hỉhh dạng trong bàng mã ASCII. Khi đó các nguyên âm thuần Việt sẽ được mã hóa theo hai phím: phím nguyên âm latinh và phím dấu nguyên âm đi kèm. 'Dấu thanh trong vần được mã hóa riêng và được đặt kèm nguyên âm dự định cho mang dấu. Sự khác dặt giữa các cài đặt cụ thề cho cách mã hóa này chì ở mức độ bằn phím vật lí, chọn kí tự nào trong bộ mâ ASCII biêu diễn cho các dấu nguyêu âm và dấu thanh. Nhược điểm chính của cách mã hóa này là tốn thời gian và số lần gõ phim đề tạo ra một chữ thuần Việt. Hơn nữa các phím dấu thường nằm vào các vị trí không thuận lợi cho việc sừ dụng khá thường xuyên. Uu điềm của nó là có thề tạo ra dạng nhỉn dể nhận biết cho chữ Việt biều diên theo ASCII 7 bit, do đó có thể dung được cho các mạng truyền thông 7 bit.

\subsubsection{Kiêu bộ mã TELEX 5 bit}

Cách mã hóa dựa trên bảng mã TELEX 5 bit bao gồm việc các nguyên âm và dấu thanh đều được mã hơa theo các chữ cái latinh theo quy định thống nhất:

$$
\begin{aligned}
& \text { dấu huyền - chữ } \mathrm{f}, \quad \text { dấu mũ - } 2 \text { lì̀n kí tự nguyên âm, } \\
& \text { dấu hòi - chữr, dấu mũ ngực - chữ } \mathrm{w} \\
& \text { dấu ngã - chữ } x, \quad \text { dấu râu } \quad \text { - chữ } w \\
& \text { dấu saes c - chũs, } \\
& \text { - dấu nặng - chữj }
\end{aligned}
$$

Dặc điêm của cách mã hóa này là giúp có được tốc độ gõ vào bàn phím rất nhanh vi việc bố trí các phím dấu đã được chọn vào các vị trí khá thuận lợi cho ngón tay gõ. Nhược điềm chính của nó là cần phải có thời gian để người sư dụng quen với cách mã hóa các con chữ Việt.

Một biến thể của cách mã hóa này người dùng gõ dấu thanh vào phần sau từ bên cạnh việc gô dấu thanh ngay sau nguyên âm mang dấu. Hơn thế nữa, người dùng có thể thay đồi các thanh trên từ mà không phải sứa nguyên âm mang dấu thanh kề cả thanh không (tức là không có dấu thanh). Khả năng logic trong việc thay đổi thanh trên tù này có thể được cài đặt cho mọi kiểu mã hóa logic chữ Việt đã trinh bày ở trên theo các bàn phím quốc tế. 


\subsubsection{Kiều chữ máy Olimpia}

('ách mã hóa theo kiêu nıáy chữ Olimpia bao gồm việc mọi chữ Việt đêu được ıà hóa thành chữ latinh cơ sở kết hợp với dấu nguyên âm găn kèm dấu thanh. Iột số phím được chọn ra để mã hóa cho tồ hợp các dấu: dấu mũ và dấu huyền, dấu mũ và dấu săc.. Cách mã hóa này ihỉ thuận tiện cho người đánh máy đã quen với loại máy chữ này. Ngoài ra, việc bî́ trí các tồ hợp dấu nguyên âm và dấu thanh là không thỏa đáng và mang nhiêu dầi ấn của các ràng buộc kí thuật từ thời các máy chữ cơ khí.

\subsubsection{Kiêu con chữ dựng sằn}

Cách mã hóa theo con chừ dựng sẵn: dùng các chức năng cài đặt ớ mức 3,4 dề tạo ra chữ Việt có mang dấu thanh. Có nghia là dùng các phím "Alt" hay "option" đi kèm theo các phím khác đề tạo chữ Việt có mang dấu. Nhược điêm lớn nhất của câch mã hóa này là tồ liợp các chừ Việt có mang dấu thanh quá lớn nên gây khó khăn cho người sử dụng trong việc ghi nhớ các phím bấm.

\section{Mã hóa theo bàn phím vật lí}

Tất cả các phương án mà hóa theo bàn phím logic trên đây đêu dáp ứng được yêu câu biểu diển đây đủ cho chữ Việt. Yêu câuu tương hợp cho các loại bàn phím quốc tế cunng được thỏa màn do việc xuất phát điêm của ta là từ các bàn phím quốc tế, bên cạnh việc bào toàn mọi tỉuh chất của bàn phím quốc tế, chúng ta chỉ tìm cách làm quá tải thêm nó đề có thề diển tả được cho các chữ Việt. Yêu cầu vê tỉnh uhất quán cùng được thóa màn vì ngoài phân đã được bảo toàn của bàn phím quốc tế, đã tuân thư các chuầı quốc tế vể bàn phím, phẩn chúng ta phát triền thêm cũng dựa trên các chuân quốc tế.

Sau khi xác định được quy tắc mà licia các chữ Việt theo các phím bấm trên bàn phím quốc tể và các quy tắc phân tích, tái tạo chữ Việt từ các tín hiệu bàn phím, vấn đề nữa cân phải để cập tới là xác đlịnh cách bố trí vật lí cho các phím đó trên bàn phím. Việc này cần được tiến hành với mục tiêu đáp ứng cho yêu cầu dễ sư dụng đối với đại đa số người dùng vốn chưa quen thuộc nhiều với tin học. Việc này mặt khác đòi hỏi phải thông qua các số liệu thống kê vể tân suất của các con chữ Việt và thanh đề xác định các tồ nguyên âm và thanh nào hay xằy ra nhất từ đó ược bố trí vào các vị trí thuận tiện uhất cho ngón tay đó.

Các số liệu thống kê về tấn suất cria các con chừ Việt được tóm tắt như sau.

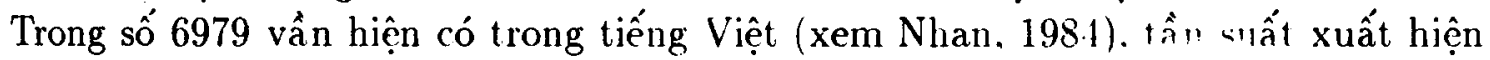
của các nguyên âm là: 


$\begin{array}{lc}\text { yê / iê / ya / ia / } & 41 \text { i }(\mathrm{ya}, \mathrm{ia}=60) \\ \hat{\text { ô }} & 518 \\ \text { ơ } & 583 \\ \text { uô / ua / uê } & 208(\mathrm{ua}=63) \\ \text { ư } & 248 \\ \text { ưa / ươ } & 303(\text { ưa }=74)\end{array}$

\section{3,847 (hoặc 3,724 nếu bỏ bớt ia, ya, ua) \\ $55.12 \% \quad 53.36 \%$}

tức là hơn một nửa các vần hiện có dều mang dấu thanh. Có thể hớt luận rằng nếu ta chấp thuận một chuần cho bàn phím có dấu nguyên âm thì số bän phím cần gõ thêm sẽ tăng lên tới $153-155 \%$.

Xem xét sơ bộ một bài báo 2135 tìr có khoạng 673 dấu mũ, 474 dấu râu và 63 dấu mũ ngược, trong số 12623 byte (hoặc gần như 6 kí tự một từ). Vậy nếu dấu nguyên âm được gõ bằng phím riêng biệt thì số lần gõ thêm sẽ chiếm 10,60\%. Mặc dầu $54 \%$ số tù̀ là có dấu nguyên âm cùng chỉ chiếm hơn $10,60 \%$ số byte trong cá bài viết.

\section{Kiến nghị về cách tổ chức bàn phím chữ Việt}

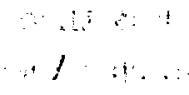

Lựa chọn định nghĩa bàn phím mới hay làm quá tải bàn phím cũ là một lựa chọn đầu tiên cân phải tính tới. Tìr các trỉnh bày ở trên chúng tôi nghiêng vể hướng làm quá tải các bàn phím đà có sắn.

Với các bàn phím hiện tại, lựa chọn tiếp theo là nên dựa trên cấu hỉnh của bàn phím QWRTY hay AZERTY. Do tínl phồ biến của bàn phím QWERTY hiện tại, câu trả lời nghiêng về QWERTY. Tuy nhiên đề vẫn có thề sừ dụng bàn phím AZERTY trong các trường hợp cân thiết, các chương trình bàn phím nên cho phép người sư dụng tự định nghîia lại tương ứng giữa các phím logic và vật lí.

Trong số 12 phím cần thêm vào cho chữ Việt, có 7 phím mô tả cho các nguyên âm thuần Việt, cần được cài đặt ở hai mức tương ứng với các chữ thường và hoa, còn 5 phím chỉ cân cài đặt ờ một mức tương ứng với 5 dấu thanh. Có thể chọn 5 vị trí trên hàng chữ số đề bố trí cho 5 dấu thanh này.

Các chữ cái tiếng Anh $\mathrm{f}, \mathrm{j}, \mathrm{w}, \mathrm{z}$ là uhững chữ hầu như không có trong các văn bản Việt nên có thể xem xét sử dụng các vị trí đó cho các nguyên âm thuân Việt. Mặt khác, cû̀ng có thể xem xét sử dụng vị trí của các dấu $[],,=$ cho các nguyên âm thuần Việt. Sau đây là một số dự kiến về cácłt bố trí bàn phím chữ Việt:

Mức 1: 


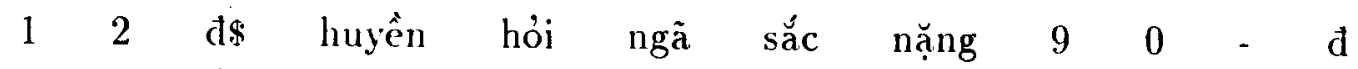

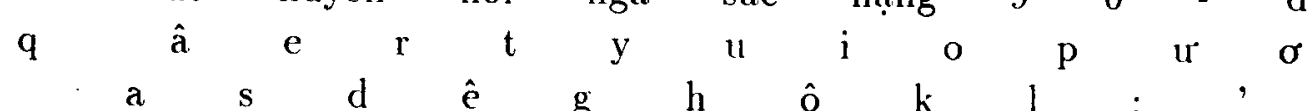
$\begin{array}{lllllllllllllll}\mathrm{a} & & \mathrm{s} & \mathrm{d} & \hat{\mathrm{e}} & \mathrm{g} & \mathrm{h} & \hat{\mathrm{o}} & \mathrm{k} & \mathrm{l} & ;\end{array}$

Mức 2 (Shift):

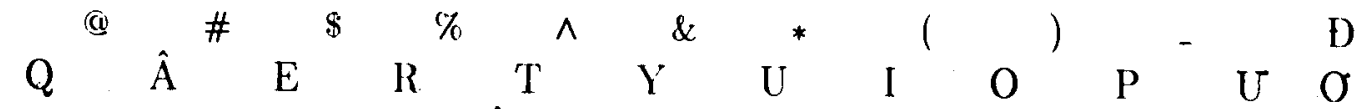

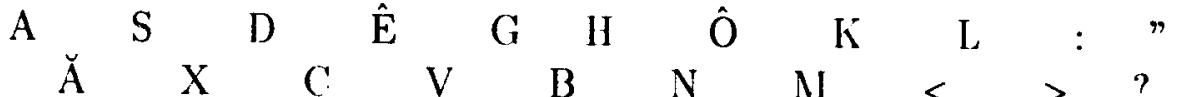

Mức 3: (Alt/Option)

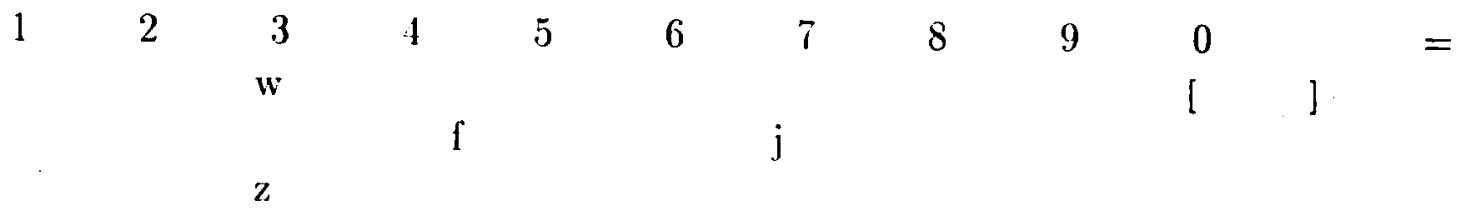

Mưc 4 (Shift + Alt / Option)

W

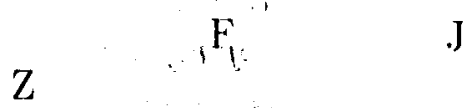

Nột dụ kiến khác vê cách bố trí bàn phím chữ Vị̣̂t ( để tránh xung dột với việc ding Alt-F, W, J, Z, trong windows)

Mức 1:

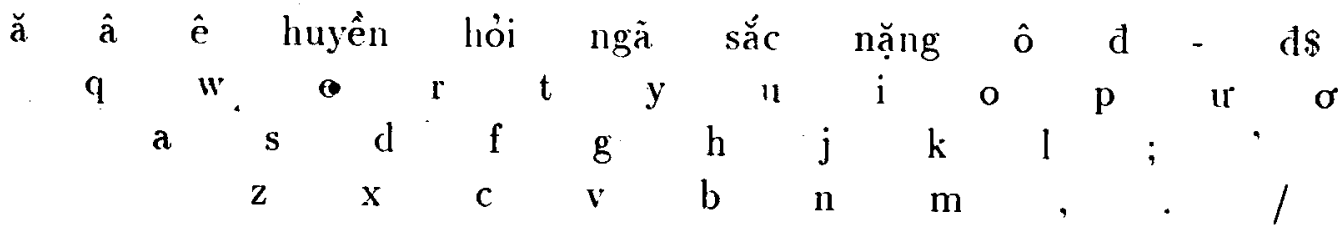

Mức 2 (Shift):

$\begin{array}{llllllllll}\check{A} & \hat{\mathrm{A}} & \hat{\mathrm{E}} & \$ & \% & \wedge & \& & * & \hat{\mathrm{O}} & \mathrm{D}\end{array}$

$$
\begin{aligned}
& \begin{array}{llllllllllllllllllllll}
\text { Q } & \text { W } & \text { E } & \text { R } & \text { T } & \text { Y } & \text { U } & \text { I } & \text { O } & \text { P } & \text { U } & \text { O }
\end{array}
\end{aligned}
$$

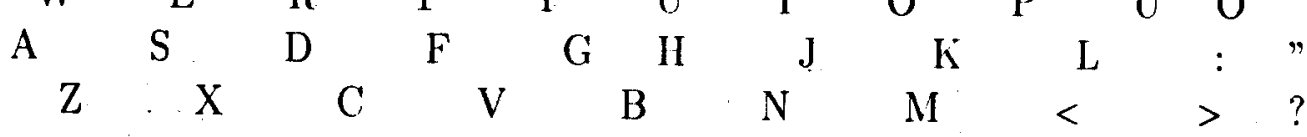

Mức 3: (Alt/Option)

$\begin{array}{lllllllllll}1 & 2 & 3 & 4 & 5 & 6 & 7 & 8 & 9 & 0 & \end{array}=$




\section{Mức 4 (Shift + Alt / Option)}

!. @ \# $\quad$ \&

\section{Tài liệu tham kháo}

1. ISO 9995-7

2. ECMA 115

3. TCVN 5712

4. VietRest, Seatic

5. BKED 6.0

6. Viet-Std: VISCII \& VIQR

7. Ngo Thanh Nhan, Ve tu bo dau chu Viet, 1984

\section{Abstract}

\section{Computer keyboard for Vietnamese}

Vietnamese keyboard i9s an indispensable tool to use Vietnamese on computers. This paper is intended to present some principals should be considered when designing kycboard for Vietnamese and some problems of its instalation. Vietnamese kyeboard should be conformed main points of view's developement of information technology in lietnam and satisfied four requirements: completentss, consistency, compatibility and sigmplicity. Based on principals of international kyeboards, according to standards ISO 9995 and EC.MA 115, and some Vietnamese particulars, methods of encoding and rendering Vietnamese characters from international kyeboards are considered and commented. Tuo solutions for designing Viftnamese kyeboard satisfying the four above requirements are proposcd. 